\title{
Immunogenicity of biological treatments in dermatology
}

\author{
Helena Iznardo and Lluís Puig* \\ Department of Dermatology, Hospital de la Santa Creu i Sant Pau, Barcelona, Spain
}

\begin{abstract}
Biological treatments have revolutionized treatment of chronic inflammatory diseases, but with them comes along a potentially relevant snag: drug immunogenicity. Being foreign proteins, biological drugs are can be immunogenic and elicit formation of anti-drug antibodies (ADA). ADA are able to decrease the efficacy of biologics through direct neutralization, blocking their binding sites, or by forming immune complexes that increase their clearance. In addition, they can also contribute to adverse immune reactions. Several factors are involved in ADA formation, both-patient and drug-related. Clinicians should be knowledgeable about drug immunogenicity, but this is a rather complex and changing field. In this comprehensive scoop we have addressed these issues, including ADA formation, methods of detection, and clinical relevance, especially focusing on management of drug immunogenicity, in psoriasis therapy. Priorities in drug immunogenicity management include identifying patients and therapies at risk for ADA development and defining preventive strategies. Patients at risk of ADA development, especially with highly immunogenic drugs, might benefit from regular monitoring of serum trough drug concentrations and $A D A$ levels, as well as concomitant treatment with immunosuppressive drugs.
\end{abstract}

Key words: Antidrug antibodies. Biologics. Immunogenicity. Psoriasis. Treatment.

\section{Introduction}

Biological treatments represent the most novel therapeutic strategy in chronic inflammatory diseases, with superior efficacy and better safety data than previous systemic therapies. They have revolutionized treatment of diverse dermatological conditions such as psoriasis, chronic urticaria, hidradenitis suppurativa (HS) and most recently, atopic dermatitis. Blockade of different cytokines implicated in the pathogenesis of immune-mediated diseases is achieved by monoclonal antibodies (mAbs). mAbs are immunoglobulins produced in cell cultures with recombinant DNA technology. Different structures are obtained according to the degree of humanization of $\mathrm{mAbs}^{1}$. Despite their immunoglobulin structure, these are foreign proteins, and thus potentially immunogenic, leading to production of anti-drug antibodies (ADA) that may neutralize their binding and activity or increase their clearance ${ }^{2}$. Most ADA are directed against the hypervariable regions of immunoglobulins (complementary-determinant regions [CDR]) forming the antigen-binding site of the therapeutic antibody (anti-idiotypic antibodies) $)^{3,4}$. ADA are predominantly high-affinity IgG isotypes ${ }^{5}$. Anti-idiotypic antibodies directed against a biologic drug compete with it for target binding, leading to neutralization of its effects. Drug immunogenicity may reduce drug efficacy through this mechanism or by formation of immune complexes that result in increased clearance, thus shortening the drug's half-life. Furthermore, adverse immune reactions can be elicited by ADA, such as infusion reactions, serum sickness, and anaphylactic reactions ${ }^{6}$. To make things more complicated, detection of ADA is not easy and several limitations exist in the assays used.

This is a comprehensive narrative review on drug immunogenicity, including ADA formation, their clinical relevance and methods of detection, with a special
Correspondence:

*Lluís Puig

E-mail: Ipuig@ @santpau.cat

2696-824X / 2021 Permanyer. This is an open access article under J CC BY-NC-ND Iicens
Accepted: 19-08-2021

DOI: $10.24875 /$ JIMIDS.M21000001
Available online: 21-10-2021

J IMIDs. 2021;1(1):2-9

www.JournalofIMIDs.com 
focus on management of drug immunogenicity in patients with psoriasis. A PubMed search was made using the terms "psoriasis" AND "immunogenicity" OR "antibodies" from start of records.

\section{ADA formation}

Factors involved in the formation of ADA can be divided roughly into patient-related and treatment-related.

Patient-related factors include genetic factors, age, previous treatments, and prior exposure to similar proteins. Although not all patients receiving the same biologic drug will develop ADA, those who develop them against a tumor necrotic factor (TNF) inhibitor will easily develop them against other biologics ${ }^{7,8}$. Genetic factors involved in patient predisposition to ADA formation have been studied. Different human leukocyte antigen (HLA) alleles are associated with formation of ADA against interferons and TNF inhibitors. HLA-DRB1 alleles are associated with formation of ADA against interferon- $B$, infliximab, and adalimumab ${ }^{9,10}$. HLA$D R B 1^{*} 03$ is independently predictive of ADA formation in rheumatoid arthritis (RA) and HS patients treated with adalimumab ${ }^{10}$. Furthermore, HLA-DQA1*05 has been associated with development of ADA in infliximaband adalimumab-treated patients with Crohn's disease $^{11}$. In this observational study, the highest rates of ADA development corresponded to patients treated with infliximab monotherapy who carried the HLA$\mathrm{DQA} 1^{*} 05$ allele had $(92 \%$ at 1 year) whereas the lowest (10\% at 1 year) corresponded to patients treated with adalimumab combination therapy who did not carry HLA-DQA $1^{*} 05^{11}$.

In addition, immune factors also play a role in ADA formation, although the exact mechanisms are not well known. The role of autoantibodies has been studied in patients with psoriasis treated with infliximab and adalimumab ${ }^{12,13}$. Hoffman et al. found an increase in median antinuclear antibody (ANA) titers from 1:80 pretreatment to $1: 280$ after the $10^{\text {th }}$ infusion, and $1: 1920$ after the $20^{\text {th }}$ infusion of infliximab. Furthermore, infliximab-ADA-positive patients and patients with loss of response had significantly higher pretreatment anti-dsDNA concentrations and higher pretreatment ANA titers than infliximab-ADA-negative and responsive patients, respectively ${ }^{12}$. Another study with 97 patients treated with anti-TNF biologics (etanercept, infliximab, and adalimumab) analyzed the development of ANA and anti-ds-DNA antibodies ${ }^{13}$. ANA developed in $17 \%$ of biologic-naïve patients, $54 \%$ of patients who had failed one treatment, $78 \%$ of patients who had failed two treatments, and $83 \%$ of patients who had failed three treatments, whereas antidsDNA antibodies developed in $2 \%, 27 \%, 33 \%$, and $83 \%$ patients, respectively ${ }^{13}$. These results suggest that, in patients being treated with anti-TNF, development of ANA and anti-dsDNA could be an indicator of future failure to further anti-TNF treatment ${ }^{13}$. Other immune mechanisms have been studied: NOTCH2 expression and increased frequency of pro-inflammatory monocyte subsets were identified as baseline predictors of ADA development in multiple sclerosis patients treated with IFN- $\beta^{14}$. Furthermore, a low frequency of signal regulatory protein (SIRP) $\alpha / \beta+$ memory $B$ cells was found in patients treated with adalimumab for RA who developed $\operatorname{ADA}^{15}$. Finally, baseline severity of disease might also play a role in ADA formation, as higher disease activity has been observed in RA patients who developed ADA $^{16}$. All these factors can influence selection of the most appropriate biologic therapy for individual patients.

Drug and therapeutic regime-related factors are also involved in ADA development ${ }^{17}$. Route of administration, dose, serum trough concentration of the drug, frequency of administration, and duration of treatment play important roles. Intravenously administered biologics are usually less immunogenic than those given through subcutaneous or intramuscular route, as the latter routes favor antigen-presenting cell uptake and presentation ${ }^{17}$. Continuous administration is usually less immunogenic than intermittent one, and higher doses given without interruption over a longer period have been associated with less immunogenic responses than intermittent lower doses. Specifically, higher doses have been associated with lower ADA rates for infliximab $^{18}$, ustekinumab ${ }^{18}$ and certolizumab ${ }^{19}$, but not with tildrakizumab ${ }^{20}$. Reexposure after a long treatment-free interval might be associated with enhanced immune response ${ }^{17}$. In fact, increased risk of infusion reactions has been associated with intermittent therapy in psoriasis: in the RESTORE2 clinical trial, patients were randomized 1:1 to continuous therapy (infliximab $5 \mathrm{mg} / \mathrm{kg}$ every 8 weeks) or intermittent therapy (no treatment until $>50 \%$ loss of PASI improvement), with $1 / 222$ $(<1 \%)$ versus $8 / 219(4 \%)$ patients, respectively, experiencing infusion-related reactions, which were associated with high titers of ADA (5/9 patients with serious infusion-related reaction had ADA titers $\geq 1: 1280)^{21}$. Interestingly, development of ADA has been reported to be transient in psoriasis patients treated with infliximab $^{22}$, adalimumab ${ }^{22,23}$, etanercept ${ }^{24}$, and brodalumab $^{25}$, suggesting that some sort of immune tolerance 
can occur ${ }^{26}$. Concomitant treatment with immunosuppressant drugs typically reduces the immunogenicity in anti-TNF treatment ${ }^{17}$. A meta-analysis with 936 patients treated with adalimumab or infliximab for RA, inflammatory bowel disease, spondyloarthritis, and psoriasis showed than concomitant methotrexate (MTX) or azathioprine/mercaptopurine reduced the detectable ADA frequency by $47 \%{ }^{27}$.

Protein structure is also relevant in ADA formation. Antibody chimerization refers to the fusion of mouse immunoglobulin variable domain regions with human immunoglobulin constant regions ${ }^{28}$. Humanized antibodies are developed by grafting mouse complementary-determining regions (CDR) - the part of the variable chains that bind to their specific antigen - into a human antibody backbone, reducing the amount of non-human sequences ${ }^{29}$. Fully human antibodies result from phage display libraries expressing human antibody fragments or from mice genetically modified to express human immunoglobulin genes ${ }^{30}$. Naturally, chimeric antibodies such as infliximab have a higher immunogenic potential than humanized or human antibodies $^{2}$. However, fully human mAbs such as adalimumab can also produce ADA that target the CDRs forming the drug binding site (anti-idiotype) ${ }^{31}$. There are three types of anti-idiotype ADA depending on the way the interact and detect biologic drugs: blocking, non-blocking and complex specific anti-idiotype ADA (Fig. 1). Likewise, bivalent mAbs are more likely to form larger immune complexes with ADA compared to fusion proteins. Etanercept is a dimeric fusion protein comprised of two extracellular portions of $75-\mathrm{kDa}$ TNF receptor, linked to the Fc portion of a human IgG1. As it does not include any CDR in its structure, etanercept has shown significantly less immunogenicity than TNF inhibitor mAbs, with higher drug survival ${ }^{32}$. ADA directed to etanercept have been described, although they appear to be non-neutralizing, not involving the TNFbinding site of the molecule ${ }^{33}$. Finally, there is also concern with ADA formation in biosimilar drugs, as stability of the product and process-related impurities and excipients can influence the immunogenic potential. However, Phase III randomized clinical trials and post-commercialization studies have reported similar ADA rates - and cross-reactivity - between reference and biosimilar drugs ${ }^{34-37}$. Furthermore, the effect of switching from the original product to biosimilars has been shown to determine no significant quantitative or qualitative changes in immunogenicity $35,38,39$. In addition, acquired experience from in silico drug design has allowed production of new biologic drugs with very low levels of immunogenicity, irrespective of their protein structure (humanized or fully human).

\section{ADA detection}

Different techniques can be used to measure ADA, with some limitations; one of the most relevant is drug interference, which limits the capacity to detect ADA due to the formation of immune complexes between the drug and its ADA. This mechanism leads to an underestimation of the incidence and titers of ADA. Two other important factors to consider when assessing different assays are sensitivity, which represents the lowest ADA concentration that can be differentiated from background, and drug tolerance, defined by the highest drug concentration than can be present in the sample without preventing ADA detection'. Drug interference can be minimized by obtaining samples just before the next dose and by treating samples with several techniques to dissociate ADA-drug immune complexes (drug-tolerant assays $)^{40}$. Some of the most commonly used techniques and their particular characteristics are: enzyme-linked immunosorbent assays (ELISA) and radioimmunoassay, able to detect free ADA but not ADA-drug immune complexes; solid-phase ELISA, highly sensitive but not specific, leading to false positives; two-site binding assay, sensitive and specific, but highly susceptible to drug interference and unable to detect IgG4 antibodies; finally, the $\mathrm{pH}$-shift anti-idiotype antigen-binding test uses acidic treatment to dissociate the ADA-drug complexes ${ }^{5}$. Apart from technical difficulties in measurement, other factors limit clinical assessment of ADA: immunogenicity data are not directly comparable across studies, as different assays report variable rates of drug interference and ADA titers vary with different measurement systems, as well as unitary measures. In addition, not all studies measure the neutralizing capacity of ADA, nor are these results directly related with clinical relevance ${ }^{2}$. Finally, immunoassays must have a proper correlation in ADA detection between originator drugs and biosimilars ${ }^{41}$. Routine drug level monitoring and ADA measurements are therefore complicated, and interpretation must be careful.

\section{ADA clinical relevance}

ADA can lead to a reduction in therapeutic efficacy and/or an increased risk of adverse events, but not all patients will experience a decrease in clinical response, so assessment of the clinical relevance of ADA is 


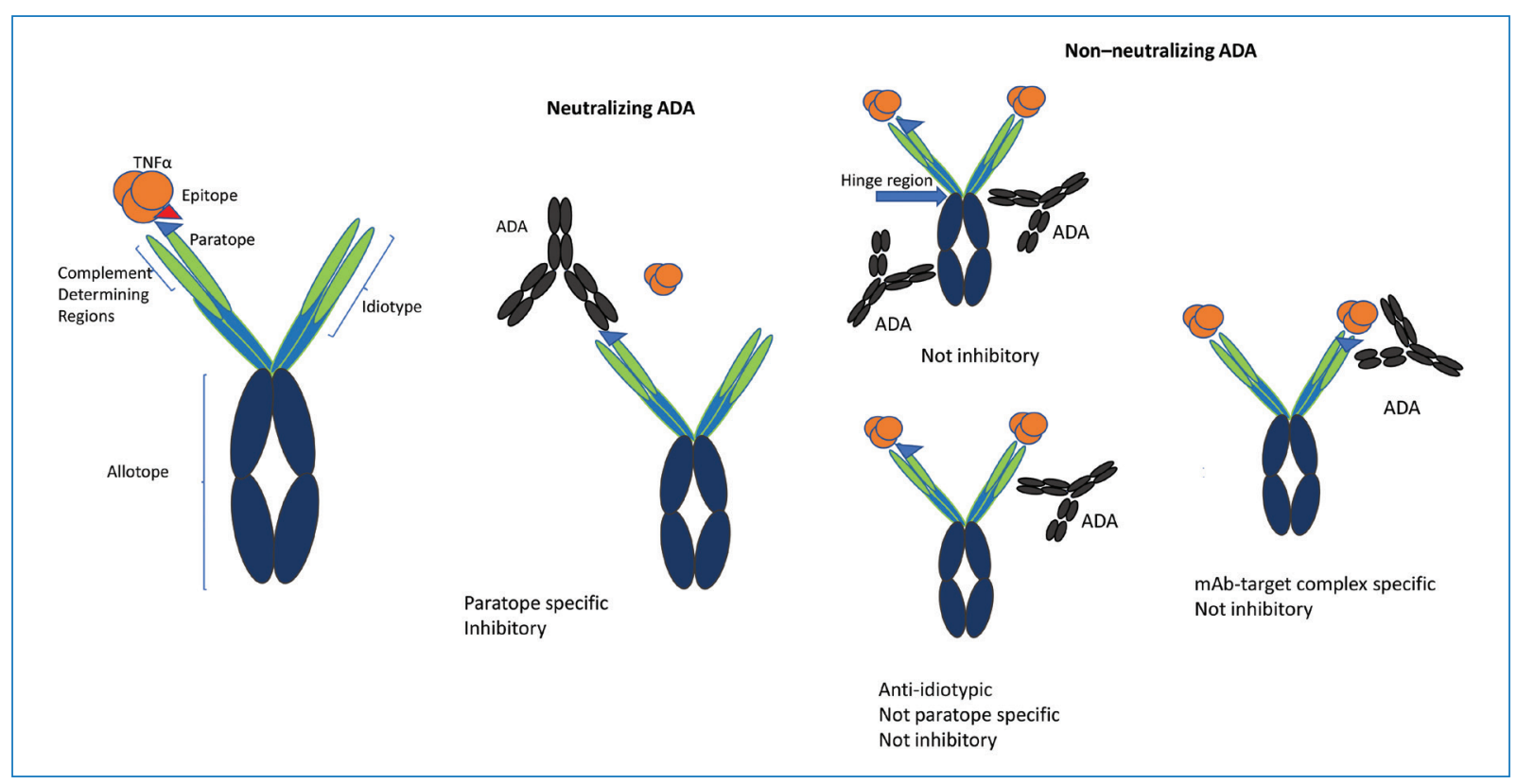

Figure 1. Neutralizing and non-neutralizing antibodies: mechanism of action. Neutralizing anti-drug antibodies (ADA) bind to paratopes in the complement determining region (either murine or human) of the $\mathrm{mAb}$, preventing fixation of the biological drug to its target, e.g. tumor necrosis factor alpha (TNF $\alpha$ ). Non-neutralizing antibodies bind to hinge regions of immunoglobulins or fusion proteins (etanercept), to idiotypes (but not paratopes), to allotopes, or to $\mathrm{mAb} /$ target complexes, thus not interfering in the ability of the biologic drug to bind to its target. Non-neutralizing ADA may reduce the efficacy of biologics through formation of immune complexes leading to increased $\mathrm{mAb}$ clearance.

important. Non-neutralizing ADA have been detected in patients treated with etanercept, without clinically relevant effects ${ }^{24}$. Low rates of ADA to secukinumab ${ }^{42}$, guselkumab ${ }^{43}$, brodalumab ${ }^{25}$, and bimekizumab ${ }^{44}$ have also been observed, without affecting efficacy. In addition, development of novel immunoassays has increased detection sensitivity of ADA compared with traditional assays, so increased rate of detection may not correlate with decreased clinical response ${ }^{45}$.

The efficacy of any biologic will be compromised below the minimally effective drug concentration, so drug levels must be evaluated in patients not experiencing or losing therapeutic response ${ }^{1}$. Other important factors include the ADA titers and whether the presence of ADA is transient or persistent (the latter are normally more clinically relevant ${ }^{27}$. Maintenance of the same mAb (or a biosimilar one) in patients who have developed clinically relevant $A D A$ will result in decreased clinical efficacy and increased ADA formation, with potential for increased frequency and severity of adverse reactions, especially hypersensitivity reactions. They tend to appear at or after the second administration and range from mild to serious infusion reactions, ranging from anaphylaxis to cytokine release syndrome and non-acute immune reactions such as immune complex disease ${ }^{17}$. Most ADA are of the $\lg G$ class, suggesting an IgE-independent pathway of anaphylatoxin production ${ }^{1}$. Increased rates of infusion reactions have been detected in patients with ADA to infliximab ${ }^{5}$.

\section{Immunogenicity data for biological treatments used in psoriasis (Table 1)}

A systematic review comparing immunogenicity of infliximab, adalimumab, secukinumab, golimumab, ustekinumab, etanercept, and abatacept across different inflammatory diseases found that infliximab and adalimumab had the highest immunogenicity rates $(80 \%$ of patients developed ADA $)^{38}$. Neutralizing ADA to infliximab and adalimumab are associated with low through drug levels, poor clinical response, increased infusion reactions, and high rate of drug discontinuation ${ }^{33}$.

Specifically for psoriasis, data from Phase III and observational studies in infliximab-treated patients show that up to $40 \%$ will develop ADA, decreasing clinical response ${ }^{21,46}$. For adalimumab, Phase III studies with Humira $\AA$ and biosimilar drug Amgevita $\AA$ found ADA 
Table 1. Summary of available data on ADAs in psoriasis

\begin{tabular}{|c|c|c|c|c|}
\hline \multirow[t]{2}{*}{ Biological drug } & \multirow[t]{2}{*}{ Molecule } & \multirow[t]{2}{*}{ ADA prevalence* } & \multicolumn{2}{|c|}{ Clinical relevance } \\
\hline & & & Efficacy & Adverse events \\
\hline Infliximab & Chimeric Mab & $27 \%$ & Yes & Yes (IR) \\
\hline Adalimumab & Humanized MAb & $9-64 \%$ & Yes & No \\
\hline Certolizumab pegol & PEGylated Fab fragment of humanized Mab & $17 \%$ & Yes & No \\
\hline Ustekinumab & Fully human Mab & $4-15 \%$ & Yes & No \\
\hline Ixekizumab & Humanized Mab & $9-17 \%$ & Yes & No \\
\hline Risankizumab & Humanized Mab & $24 \%$ & Yes & Yes (ISR) \\
\hline Tildrakizumab & Humanized Mab & $7-8 \%$ & Yes & No \\
\hline Etanercept & Fusion protein & $0-18 \%$ & No & No \\
\hline Secukinumab & Fully human Mab & $<0.4 \%$ & No & No \\
\hline Guselkumab & Humanized Mab & $9 \%$ & No & No \\
\hline Brodalumab & Fully human Mab & $2 \%$ & No & No \\
\hline Bimekizumab & Humanized Mab & $12.8 \%$ & No & No \\
\hline
\end{tabular}

ADAs: antidrug antibodies; Mab: monoclonal antibody; IR: infusion reaction; ISR: injection-site reaction. ${ }^{*}$ ADA prevalence data taken from Tsakok et al. ${ }^{2}$ and Glatt et al. ${ }^{44}$

development in up to $72.3 \%$ of patients, with similar rates for both drugs ${ }^{39}$. Another Phase III study comparing Humira $\circledast$ with Hyrimoz $®$ found ADA in $37 \%$ and $34 \%$ of patients treated with Humira $\circledast$ and Hyrimoz $\circledast$, respectively, with a similar proportion of neutralizing antibodies $(80 \%)$ by week $17^{35}$. Furthermore, patients treated initially with Hyrimoz ${ }^{\circledR}$ and then switched to Humira ${ }^{\circledR}$ reported similar rates of ADA than those treated from Humira $囚$ from the start of the study $(46.7 \%$ vs. $45.1 \%$, respectively $)^{35}$. A recent study of psoriasis patients receiving adalimumab and MTX (10 mg orally/week) has shown that concomitant treatment reduced development of $\mathrm{ADA}^{47}$. Regarding certolizumab-pegol, data from a Phase II study reported ADA development in $18 \%$ and $25 \%$ of psoriasis patients treated with $200 \mathrm{mg}$ and $400 \mathrm{mg}$ twice weekly by week $24^{48}$. Pooled data from Phase III studies CIMPASI-1 and CIMPASI-2 reported a $17 \%$ rate of ADA development, with the lower drug levels and reduced clinical responses ${ }^{19}$. ADA to ustekinumab have also been detected in psoriasis patients, with lower serum drug concentrations and lower efficacy found in these patients ${ }^{33}$. ADA rates varied between $3.5-8.7 \%$ in 2 real-world studies ${ }^{49,50}, 15 \%$ in a randomized clinical trial using a more drug-tolerant assay, 3.8\% (using a standard assay), and $10.6 \%$ (using a drug-tolerant assay) in a recent study of 340 psoriasis patients ${ }^{51}$. Interestingly, pharmacokinetic/pharmacodynamic studies have shown increased ustekinumab clearance in patients with detectable ADA, suggesting that clinical relevance of ustekinumab ADA was due to this mechanism ${ }^{52}$. Phase III studies of ixekizumab using a drug-tolerant ADA assay showed that $17 \%$ of patients developed ADA, $14 \%$ of them with high-titers and lower PASI reductions from baseline ${ }^{53,54}$. Risankizumab studies have shown that up to $24 \%$ of patients developed ADA, with reduced clinical response in only $1 \%$ of patients who developed high-titer ADA. In addition, ADA-positive patients presented a higher risk of developing injection site reactions ${ }^{55}$. Pooled data from Phase II and Phase III studies with tildrakizumab-treated patients showed that $8 \%$ of them developed ADA, which were neutralizing in $40 \%$ of cases; patients who developed ADA had increased drug clearance and decreased clinical response compared to patients without $A D A^{20}$.

ADA formation has been considered relatively low and probably not clinically relevant for some biologicals $^{2}$. Non-neutralizing ADA have been detected in up to $18 \%$ of psoriasis patients treated with etanercept, with no effect on clinical efficacy ${ }^{24}$. ADA to guselkumab have been detected in $9 \%$ of psoriasis patients in Phase III randomized clinical trials, with no reported impact on drug levels or clinical response ${ }^{43}$. Phase III studies have shown that $<1 \%$ of patients treated with secukinumab developed $A D A$, with no reported impact 
on clinical response ${ }^{42}$. The immunogenicity of brodalumab has also been assessed: $2.5 \%$ of 4,461 patients developed non-neutralizing ADA without clinical relevance, and they were transient in more than half of those cases $(1.4 \%)^{25}$. Finally, in a study with 39 patients treated with bimekizumab, ADA were detected in five subjects by week 20 , with no reported effect on pharmacodynamic parameters ${ }^{44}$.

\section{Clinical management of ADA}

So far consensus clinical guidelines do not include a common approach to ADA management, although patients and clinicians would benefit extensively from one, especially in those specialties where therapeutic options are limited or the clinical efficacy of biologic treatment cannot be assessed visually. In a cohort of 105 RA patients receiving biologics, immunogenicity assessment was associated with higher probabilities of achieving response and lower disease activity ${ }^{56}$. Carrascosa et $\mathrm{al}^{33}$. have proposed a pragmatic algorithm regarding drug immunogenicity: in patients with loss of clinical response or suboptimal responses after an initial good response, monitoring of serum drug through levels can be helpful. If adequate drug levels are present, consideration should be given to switching to another biologic with a different mechanism of action. On the contrary, when suboptimal drug levels are detected, measurement of ADA levels can be helpful in deciding to switch to a different TNF inhibitor in ADApositive patients and increasing drug dose or dosing frequency in ADA-negative ones.

\section{Conclusions}

With the increasing use of biologic drugs and biosimilars on a daily basis, clinicians must be familiarized with drug immunogenicity. However, as we have seen, several difficulties exist in assessing ADA - especially regarding detection assays - and deciding on their clinical relevance. Basic knowledge of the different assays and common terminology is needed when evaluating a suspicious case of drug immunogenicity. In addition, drug levels should always accompany ADA data, since reductions in clinical efficacy are commonly mediated through reduced drug exposure. Furthermore, the risk of ADA development and their clinical implications vary among different treatments. Thus, management of drug immunogenicity frequently requires an individualized assessment. To establish a common approach, a recent categorization in three groups can be helpful: Group 1 includes biologics more likely to develop clinically relevant ADA, such as adalimumab and infliximab; Group 2 comprises drugs associated with lower risk of developing clinically relevant ADA, namely ustekinumab, tildrakizumab, risankizumab, and ixekizumab; Group 3 includes those biologics for which development of clinically relevant ADA has not been reported to date: etanercept, secukinumab, guselkumab, and brodalumab ${ }^{2}$. Patients under treatment with Group 1 drugs (adalimumab or infliximab) could benefit from the addition of concomitant treatment with immunosuppressive drugs such as MTX, although more research is needed regarding dosing, timing of initiation, and drug of choice. Regular monitoring of ADA titers and drug levels in these patients might also contribute to rationalize drug administration, modifying treatment early on the appearance of clinically relevant ADA or hypersensitivity reactions. For patients in Group 2, further research is needed to identify potential individual factors that might increase their susceptibility to ADA development. The association between genetic and immune predisposing factors (i.e. preexisting ADA and ANA) with ADA formation has been studied, and could be assessed in routine clinical practice. These patients would probably benefit from less immunogenic biologic drugs or concomitant treatment with immunosuppressive drugs. Development of comparable assays with increased sensitivity for ADA-drug immune complexes and irrespective of drug levels, will contribute to our better understanding of the mechanisms of immunogenicity. In conclusion, identifying patients and therapies at risk for ADA development and defining preventive strategies, taking always into account their clinical relevance, are research priorities for new and existing biologic therapies.

\section{Funding}

None.

\section{Conflicts of interest}

H. Iznardo has perceived speaker's honoraria from Amgen. L. Puig has perceived consultancy/speaker's honoraria from and/or participated in clinical trials sponsored by Abbvie, Almirall, Amgen, Baxalta, Biogen, Boehringer Ingelheim, Celgene, Gebro, Janssen, JS BIOCAD, Leo-Pharma, Eli Lilly and Co., Merck-Serono, MSD, Mylan, Novartis, Pfizer, Regeneron, Roche, Sandoz, Samsung-Bioepis, Sanofi, and UCB. 


\section{Ethical disclosures}

Protection of human and animal subjects. The authors declare that no experiments were performed on humans or animals for this study.

Confidentiality of data. The authors declare that no patient data appear in this article.

Right to privacy and informed consent. The authors declare that no patient data appear in this article.

\section{References}

1. Garcês S, Demengeot J. The immunogenicity of biologic therapies. Curr Probl Dermatol. 2018;53:37-48.

2. Tsakok T, Rispens T, Spuls P, Nast A, Smith C, Reich K. Immunogenicity of biologic therapies in psoriasis: myths, facts and a suggested approach. J Eur Acad Dermatol Venereol. 2021;35:329-37.

3. Harding FA, Stickler MM, Razo J, DuBridge RB. The immunogenicity of humanized and fully human antibodies: residual immunogenicity resides in the CDR regions. MAbs. 2010;2:256-65.

4. van Schie KA, Hart MH, de Groot ER, Kruithof S, Aarden LA, Wolbink GJ et al. The antibody response against human and chimeric anti-TNF therapeutic antibodies primarily targets the TNF binding region. Ann Rheum Dis. 2015;74:311-4

5. Jullien D, Prinz JC, Nestle FO. Immunogenicity of biotherapy used in psoriasis: the science behind the scenes. J Invest Dermatol. 2015; 135:31-8

6. Maggi E, Vultaggio A, Matucci A. Acute infusion reactions induced by monoclonal antibody therapy. Expert Rev Clin Immunol. 2011; 7:55-63.

7. Bartelds GM, de Groot E, Nurmohamed MT, Hart MH, van Eede PH Wijbrandts CA, et al. Surprising negative association between IgG1 allotype disparity and anti-adalimumab formation: a cohort study. Arthritis Res Ther. 2010;12:R221.

8. Chirmule N, Jawa V, Meibohm B. Immunogenicity to therapeutic proteins: impact on PK/PD and efficacy. AAPS J. 2012;14:296-302.

9. Billiet T, Vande Casteele N, Van Stappen T, Princen F, Singh S, Gils A, et al. Immunogenicity to infliximab is associated with HLA-DRB1. Gut. 2015;64:1344-5.

10. Liu M, Degner J, Davis JW, Idler KB, Nader A, Mostafa NM, et al. Identification of HLA-DRB1 association to adalimumab immunogenicity. PLoS One. 2018;13:e0195325

11. Sazonovs A, Kennedy NA, Moutsianas L, Heap GA, Rice DL, Reppell M, et al. HLA-DQA $1{ }^{\star} 05$ carriage associated with development of anti-drug antibodies to infliximab and adalimumab in patients with Crohn's disease. Gastroenterology. 2020;158:189-99.

12. Hoffmann JH, Hartmann M, Enk AH, Hadaschik EN. Autoantibodies in psoriasis as predictors for loss of response and anti-infliximab antibody induction. Br J Dermatol. 2011;165:1355-8.

13. Pink AE, Fonia A, Allen $\mathrm{MH}$, Smith $\mathrm{CH}$, Barker JN. Antinuclear antibodies associate with loss of response to antitumour necrosis factor-alpha therapy in psoriasis: a retrospective, observational study. $\mathrm{Br} \mathrm{J}$ Dermatol. 2010;162:780-5

14. Adriani M, Nytrova P, Mbogning C, Hässler S, Medek K, Jensen PE, et al Monocyte NOTCH2 expression predicts IFN- $\beta$ immunogenicity in multiple sclerosis patients. JCI Insight. 2018;3:99274.

15. Magill L, Adriani M, Berthou V, Chen K, Gleizes A, Hacein-Bey-Abina S, et al. Low percentage of signal regulatory protein $\alpha / \beta+$ memory $B$ cells in blood predicts development of anti-drug antibodies (ADA) in adalimumab-treated rheumatoid arthritis patients. Front Immunol. 2018:9:2865.

16. Bartelds GM, Krieckaert CL, Nurmohamed MT, van Schouwenburg PA, Lems WF, Twisk JW, et al. Development of antidrug antibodies against adalimumab and association with disease activity and treatment failure during long-term follow-up. JAMA. 2011;305:1460-8.

17. European Medicines Agency. Guideline on Immunogenicity Assessment of Biotechnology-derived Therapeutic Proteins. Amsterdam, Netherlands: European Medicines Agency; 2007. p. 18.

18. Jullien D. Anti-drug antibodies, auto-antibodies and biotherapy in psoriasis. Ann Dermatol Venereol. 2012;139:S58-67.

19. Cymzia Product Information. Cimzia 200mg Solution for Injection in Pre-Filled Syringe-summary of Product Characteristics (SmPC)-(emc). Available from: https://www.medicines.org.uk/emc/product/4450/smpc. [Last accessed on $2021 \mathrm{Jul} 31$ ].

20. Kimball AB, Kerbusch $T$, van Aarle F, Kulkarni $P$, Li Q, Blauvelt $A$, et al Assessment of the effects of immunogenicity on the pharmacokinetics, efficacy and safety of tildrakizumab. Br J Dermatol. 2020;182:180-9.
21. Reich K, Wozel G, Zheng H, van Hoogstraten HJ, Flint L, Barker J. Efficacy and safety of infliximab as continuous or intermittent therapy in patients with moderate-to-severe plaque psoriasis: results of a randomized, long-term extension trial (RESTORE2). $\mathrm{Br} J$ Dermatol. 2013:168:1325-34

22. Ben-Horin S, Mazor Y, Yanai H, Ron Y, Kopylov U, Yavzori M, et al. The decline of anti-drug antibody titres after discontinuation of anti-TNFs: mplications for predicting re-induction outcome in IBD. Aliment Pharmacol Ther. 2012;35:714-22.

23. Menting SP, van Lümig PP, de Vries $A C$, van den Reek JM, van der Kleij $D$, de Jong EM, et al. Extent and consequences of antibody formation against adalimumab in patients with psoriasis: one-year follow-up. JAMA Dermatol. 2014;150:130-6

24. Tyring S, Gordon KB, Poulin Y, Langley RG, Gottlieb AB, Dunn M, et al. Long-term safety and efficacy of $50 \mathrm{mg}$ of etanercept twice weekly in patients with psoriasis. Arch Dermatol. 2007;143:719-26.

25. Iznardo $\mathrm{H}$, Puig L. The safety of brodalumab for the treatment of psoriasis. Expert Opin Drug Saf. 2020;19:365-72.

26. van Schouwenburg PA, Rispens T, Wolbink GJ. Immunogenicity of anti-TNF biologic therapies for rheumatoid arthritis. Nat Rev Rheumatol. 2013;9:164-72.

27. Garcês S, Demengeot J, Benito-Garcia E. The immunogenicity of anti-TNF therapy in immune-mediated inflammatory diseases: a systematic review of the literature with a meta-analysis. Ann Rheum Dis. 2013;72:1947-55.

28. Boulianne GL, Hozumi N, Shulman MJ. Production of functional chimaeric mouse/human antibody. Nature. 1984;312:643-6.

29. Kim JH, Hong HJ. Humanization by CDR grafting and specificity-determining residue grafting. Methods Mol Biol. 2012;907:237-45.

30. Lonberg N. Fully human antibodies from transgenic mouse and phage display platforms. Curr Opin Immunol. 2008;20:450-9.

31. van Schouwenburg $P A$, van de Stadt $L A$, de Jong $R N$, van Buren $E E$, Kruithof S, de Groot E, et al. Adalimumab elicits a restricted anti-idiotypic antibody response in autoimmune patients resulting in functional neutralisation. Ann Rheum Dis. 2013;72:104-9.

32. de Vries MK, van der Horst-Bruinsma IE, Nurmohamed MT, Aarden LA Stapel SO, Peters MJ, et al. Immunogenicity does not influence treatment with etanercept in patients with ankylosing spondylitis. Ann Rheum Dis. 2009;68:531-5.

33. Carrascosa JM, van Doorn MB, Lahfa M, Nestle FO, Jullien D, Prinz JC. Clinical relevance of immunogenicity of biologics in psoriasis: implications for treatment strategies. J Eur Acad Dermatol Venereol. 2014;28:1424-30.

34. Griffiths CE, Thaçi D, Gerdes S, Arenberger P, Pulka G, Kingo K, et al. The EGALITY study: a confirmatory, randomized, double-blind study comparing the efficacy, safety and immunogenicity of GP2015, a proposed etanercept biosimilar, vs. the originator product in patients with moderate-to-severe chronic plaque-type psoriasis. $\mathrm{Br} \mathrm{J}$ Dermatol. 2017;176:928-38.

35. Blauvelt A, Lacour JP, Fowler JF, Weinberg JM, Gospodinov D, Schuck E, et al. Phase III randomized study of the proposed adalimumab biosimilar GP2017 in psoriasis: impact of multiple switches. Br J Dermatol. 2018;179:623-31.

36. Fiorino G, Ruiz-Argüello MB, Maguregui A, Nagore D, Correale C, Radice $\mathrm{S}$, et al. Full interchangeability in regard to immunogenicity between the infliximab reference biologic and biosimilars CT-P13 and SB2 in inflammatory bowel disease. Inflamm Bowel Dis. 2018;24:601-6.

37. Ruiz-Argüello MB, Maguregui A, Ruiz Del Agua A, Pascual-Salcedo D, Martínez-Feito A, Jurado $T$, et al. Antibodies to infliximab in Remicade-treated rheumatic patients show identical reactivity towards biosimilars. Ann Rheum Dis. 2016;75:1693-6.

38. Strand V, Balsa A, Al-Saleh J, Barile-Fabris L, Horiuchi T, Takeuchi T, et al. Immunogenicity of biologics in chronic inflammatory diseases: a systematic review. BioDrugs. 2017;31:299-316.

39. Papp K, Bachelez H, Costanzo A, Foley P, Gooderham M, Kaur P, et al. Clinical similarity of the biosimilar ABP 501 compared with adalimumab after single transition: long-term results from a randomized controlled, double-blind, 52-week, Phase III trial in patients with moderate-to-severe plaque psoriasis. Br J Dermatol. 2017;177:1562-74.

40. Bloem K van Leeuwen A, Verbeek G, Nurmohamed MT, Jan Wolbink $G$, van der Kleij D, et al. Systematic comparison of drug-tolerant assays for anti-drug antibodies in a cohort of adalimumab-treated rheumatoid arthritis patients. J Immunol Methods. 2015;418:29-38.

41. Neveu B, Kunst A, Prosser $C$, Robitaille R. An in vitro comparison of four different immunoassays for the monitoring of infliximab biosimilars drug levels. Clin Biochem. 2020;78:58-62.

42. Reich $\mathrm{K}$, Blauvelt $A$, Armstrong $A$, Langley RG, Fox $T$, Huang J, et al. Secukinumab, a fully human anti-interleukin-17A monoclonal antibody, exhibits minimal immunogenicity in patients with moderate-to-severe plaque psoriasis. Br J Dermatol. 2017:176:752-8.

43. Zhu Y, Marini JC, Song M, Randazzo B, Shen YK, Li S, et al. Immunogenicity of guselkumab is not clinically relevant in patients with moderate-to-severe plaque psoriasis. J Invest Dermatol. 2019;139:1830-4. 
44. Glatt S, Helmer E, Haier B, Strimenopoulou F, Price G, Vajjah P, et al. First-in-human randomized study of bimekizumab, a humanized monoclonal antibody and selective dual inhibitor of IL-17A and IL-17F, in mild psoriasis. Br J Clin Pharmacol. 2017;83:991-1001.

45. Heron CE, Ghamrawi RI, Balogh EA, Feldman SR. Immunogenicity of biologic and biosimilar therapies for psoriasis and impact of novel immunoassays for immunogenicity detection. Am $\mathrm{J}$ Clin Dermatol. 2021;22:221-31.

46. Ara-Martín M, Pinto PH, Pascual-Salcedo D. Impact of immunogenicity on response to anti-TNF therapy in moderate-to-severe plaque psoriasis: results of the PREDIR study. J Dermatolog Treat. 2017;28:606-12.

47. Van Der Kraaij G, Busard C, Van Den Reek J, Menting S, Van Bezooijen S-J, De Jong E, et al. Adalimumab Monotherapy vs. Adalimumab and Methotrexate Combination Therapy in Psoriasis: firstyear Results on Drug Survival, Effectiveness, Safety and Immunogenicity. London, United Kingdom: Cochrane Library; 2019.

48. Reich K, Ortonne JP, Gottlieb AB, Terpstra IJ, Coteur G, Tasset C, et al. Successful treatment of moderate to severe plaque psoriasis with the PEGylated Fab' certolizumab pegol: results of a phase II randomized placebo-controlled trial with a re-treatment extension. $\mathrm{Br} J$ Dermatol. 2012;167:180-90.

49. Tsakok T, Wilson N, Dand N, Loeff FC, Bloem K, Baudry D, et al. Association of serum ustekinumab levels with clinical response in psoriasis. JAMA Dermatol. 2019;155:1235-43.
50. De Keyser $\mathrm{E}$, Busard $\mathrm{Cl}$, Lanssens $\mathrm{S}$, Meuleman L, Hutten BA, Costanzo A, et al. Clinical consequences of antibody formation, serum concentrations, and HLA-Cw6 status in psoriasis patients on ustekinumab. Ther Drug Monit. 2019;41:634-9.

51. Loeff FC, Tsakok T, Dijk L, Hart MH, Duckworth M, Baudry D, et al. Clinical impact of antibodies against ustekinumab in psoriasis: an observational, cross-sectional, multicenter study. J Invest Dermatol. 2020;140:2129-37.

52. Pan S, Tsakok T, Dand N, Lonsdale DO, Loeff FC, Bloem K, et al. Using real-world data to guide ustekinumab dosing strategies for psoriasis: a prospective pharmacokinetic-pharmacodynamic study. Clin Transl Sci. 2020;13:400-9.

53. Gordon KB, Blauvelt A, Papp KA, Langley RG, Luger T, Ohtsuki M, et al. Phase 3 trials of ixekizumab in moderate-to-severe plaque psoriasis. N Engl J Med. 2016;375:345-56.

54. Reich K, Jackson K, Ball S, Garces S, Kerr L, Chua L, et al. Ixekizumab pharmacokinetics, anti-drug antibodies, and efficacy through 60 weeks of treatment of moderate to severe plaque psoriasis. J Invest Dermatol. 2018;138:2168-73.

55. Skyrizi BL. European Medicines Agency; 2019. Available from: https:// www.ema.europa.eu/en/medicines/human/EPAR/skyrizi. [Last accessed on 2021 Aug 07.

56. Garcês S, Antunes M, Benito-Garcia E, da Silva JC, Aarden L, Demengeot J. A preliminary algorithm introducing immunogenicity assessment in the management of patients with RA receiving tumour necrosis factor inhibitor therapies. Ann Rheum Dis. 2014;73:1138-43. 